\title{
Zur Constitution der Chinaalkaloide
}

\author{
(v. Mittheilung) \\ von
}

\section{Zd. H. Skraup und J. Würstl.}

Aus dem chemischen Institute der k. k. Universität in Graz.

(Vorgelegt in der Sitzung am 11. April 1889.)

Bei vergleichender Untersuchung der Oxydationsproducte, die aus den vier wichtigeren Chinaalkaloiden, dem Cinchonin, Chinin, Cinchonidin und Chinidin entstehen, hat sich gezeigt, dass die ersten zwei bei ihrem Zerfalle zwei verschiedene Chinolinderivate, die Cinchoninsäure, beziiglich Chininsäure, sonst aber dieselben Spaltungsproducte, das Cincholoipon $\mathrm{C}_{9} \mathrm{H}_{17} \mathrm{NO}_{2}$ und die Cincholoiponsäure $\mathrm{C}_{8} \mathrm{H}_{13} \mathrm{NO}_{4}$, liefern, demnach in ihrer Constitution nur sehr wenig abweichen können.

Beim Cinchonidin und Chinidin liegen ähnliche Erfahrungen auch schon vor, doch nicht im wünschenswerthen Ausmasse; denn es ist zwar gelungen, aus beiden neben Chinolincarbonsäuren, die identisch sind mit jenen, die aus Cinchonin, beziehlich Chinin entstehen, die Cincholoiponsäure darzustellen, nicht aber ein Spaltungsproduct mit neun Kohlenstoffatomen, das dem Cincholoipon aus Cinchonin und Chinidin analog: wäle, und desshalb sind die Beziehungen zwischen Cinchonidin und Chinidin nicht in dem Masse festgestellt, wie jene zwischen Cinchonin und Chinin.

Die Ausfüllung dieser Lücke, die auch das Verhältniss zwisehen den isomeren Chinaalkaloiden nahe berihrt, ist Gegenstand dieser Mittheilung.

\section{Chinidin.}

Wie der eine von uns vor einiger Zeit ${ }^{1}$ mitgetheilt hat, erhielt er aus dem Chinidin neben dem in Alkohol unlöslichen Bleisalz

1 Monatsshefte f. Chem. X, I, 65. 
der Cincholoiponsäure einen alkohollöslichen Theil, aus welchem Quecksilberchlorid noch kleine Quantitäten von Chininsäure abschied und der dann allmälig zu krystallisiren begann.

Nach mehrwöchentlichem Stehen hatten sich nun reichliche Mengen kleiner Krystallkörner gebildet, die mit einem Gemisch von Alkohol und Salzsäure angerührt, von der Mutterlauge leicht zu trennen waren, welche wieder eingedampft eine zweite mit der ersten identische Krystallisation gab. Die erste Krystallisation betrug $3 g$, die zweite $2 g$.

Durch wiederholtes Umkrystallisiren aus verdünnter Salzsäure gereinigt, bildet die Substanz compacte Prismen, die bei 199-201 ${ }^{\circ}$ unter Gasentwicklung schmelzen, chlorhältig sind und, was Ansehen und Löslichkeit betrifft, mit dem Cincholoiponchlorhydrat vollständig übereinstimmen.

$0.2357 \mathrm{~g}$ bei $100^{\circ}$ getrocknet gaben $0.162 \mathrm{Ag} \mathrm{Cl}$.

$$
\text { Cl. ....16.96 } \quad \frac{\text { Gefunden }}{17 \cdot 10}
$$

Die krystallographische Untersuchung zeigte, dass dieselben Formen auftreten wie bei dem Cincholoiponchlorhydrat aus Chinin und Cinchonin.

Beim Kochen mit Essigsäureanhydrid entstand ein Acetylderivat rom Schmelzpunkt $122-123^{\circ}$, das, schwer in kaltem, leicht in heissem Wasser löslich, beim langsamen Abdunsten grosse und gut ausgebildete Krystalle lieferte.

Durch Fällen des Chlorhydrates mit Goldchlorid schied sich die charakteristische Golddoppelverbindung in Form von dünnen, glänzend gelben Blättchen aus. Sie sind leicht in warmem, schwierig dagegen in kaltem Wasser löslich; ihr Schmelzpunkt ist $203-204^{\circ}$.

$0 \cdot 197 \mathrm{~g}$ Substanz gaben $0.0765 \mathrm{Au}$.

$$
\text { Au...... } \frac{\text { Gefunden }}{38 \cdot 32} \quad \frac{\text { Berechnet }}{38 \cdot 43}
$$

Auch die optische Untersuchung ergab Übereinstimmung: mit dem Cincholoiponchlorhydrat aus Cinchonin. Die Drehung: ist äusserst gering, wenn überhaupt vorhanden. 
Eine $8 \%$-ige Lösung von $d=1 \cdot 0158$ zeigte bei $l=200$ and $t=20$ eine Ablenkung von $\alpha_{D}=-2 \cdot 5^{\prime}$.

Skraup fand für die Substanz aus Cinchonin bei einer $2 \cdot 5 \%$-igen Lösung $\alpha_{D}=-13^{\prime}$. Der Unterschied liegt innerhalb der Fehlergrenzen, und hiemit ist die völlige Übereinstimmung des Cincholoipons ans Chinidin mit dem aus Cinchonin und Chinin ausser Zweifel. Da das Chinidin, das zu diesen Versuchen diente, nach allen Reactionen ${ }^{2}$ rein war, überdies die Ausbeute an Cincholoipon eine relativ bedeutende ist, ist es sicher, dass letzteres thatsächlich ein Derivat des Chinidins und nicht aus einer Verunreinigung des Alkaloids entstanden ist. Es kann auch weiter keinem Zweifel unterliegen, dass das Cincholoipon aus jenen Antheilen des Chinidins gebildet wurde, die sich der ersten Oxydation mit Kaliumpermanganat ${ }^{2}$ entzogen haben. Warum diesmal die Isolirung um so viel leichter war, als in anderen Fällen, vermögen wir nicht zu erklären.

\section{Cinchonidin.}

Schniderschitsch ${ }^{3}$ hat vor einiger Zeit gezeigt, dass bei der Oxydation des Cinchonidins, beziehungsweise Cinchotenidins, Cincholoiponsäure entstehe, und dass neben dem Bleisalze dieser Säure auch alkohollösliche Oxydationsproducte auftreten.

Um grössere Mengen derselben zu gewinnen, schritten wir zu einer newen Oxydation des Cinchonidins, bei welcher $200 \mathrm{~g}$ der Base ohne vorhergehende Oxydation mit Kaliumpermanganat nach der von Skraup für das Cinchonin gelieferten Vorschrift" direct mit Chromsäure oxydirt wurden.

Die Zerlegung jener Fraction des Syrups, der Cincholoipon, beziehungsweise ein analoges Spaltungsproduct enthalten musste, geschah mit Quecksilberchlorid in derselben Art, wie es Skraup für die Gewinnung des Cincholoipons aus den Oxydationsproducten des Chinins kurz beschrieben hat.

Es gelang nach ebenso Zeit wie Geduld in Anspruch nehmenden Fällungen und Auskochungen der Quecksilbersalze

1 Nonatshefte IX, 809.

2 Ebend. $X, 66$.

3 Ebend. X, 51.

4 Sitzungsber. d. kais. Akad., Abth. II, Jahrg. 1879, Juliheft. 
gegen $25 \mathrm{~g}$ einer Doppelverbindung zu isoliren, die, in warmem Wasser ungemein leicht löslich, in blätterförmigen Krystallen anschiesst und nicht glatt bei $140^{\circ}$ unter heftiger Gasentwicklung schmilzt, also die Eigenschaften des rohen Cincholoipondoppelsalzes besitzt. Das Salz in heissem Wasser gelöst, mit Schwefelwasserstoff zerlegt und bis zum dünnen Syrup eingedampft, gab nach dem Einsäen von Cincholoiponchlorhydrat in wenigen Tagen kleine Kryställchen, die durch Aufstreichen auf Thonplatten von der zähen Mutterlauge getrenut und dann aus verdünnter Salzsäure umkrystallisirt, nicht ganz $1 \mathrm{~g}$ betrugen; sie waren nach Schmelzpunkt $\left(200^{\circ}\right)$ und anderen Eigenschaften zweifellos Cincholoiponchlorhydrat.

In grösserer Menge erhielten wir dieselbe Substanz aus jenen syrupösen Quecksilberverbindungen, die aus den ersten Fällungen mit festem Quecksilberchlorid beim Auskochen mit sehr verdünnter Salzsäure in Lösung gingen. Diese syrupösen Doppelsalze, die bei wochenlangem Stehen und trotz aller Versuche, Krystallisation anzuregen, völlig amorph blieben, wurden mit Schwefelwasserstoff zerlegt, kochend mit Bleicarbonat gesättigt, eingedampft und systematisch mit Alkohol behandelt, wobei nicht unerhebliche Mengen von cincholoiponsaurem Blei als in Alkohol unlöslich ausfielen, während die Hauptmenge in Alkohol gelöst blieb. Letztere ist nach dem Verjagen des Alkohols mit Quecksilberchloridlösung mehrmals ausgefällt und das Filtrat nach dem Eindampfen wiederholt mit Quecksilberchloridlösung aufgenommen worden, so lange in kaltem Wasser schwer lösliche Doppelverbindungen zurückblieben; dann mit Schwefelwasserstoff zerlegt und eingeengt, krystallisirten nach dem Einsäen reichlich Krystalle von Cincholoiponchlorhydrat aus, deren Menge in unreinem Zustande gegen $4 g$ betrug. Nach wiederholtem Um krystallisiren aus verdünnter Salzsäure betrug die Gesammtmenge von ganz reinem Cincholoiponchlorhydrat aus Cinchonidin $2 \cdot 5 \mathrm{~g}$.

$0 \cdot 1775 \mathrm{~g}$ gaben $0 \cdot 096 \mathrm{Ag} \mathrm{Cl}$.

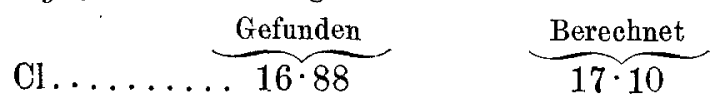

Das Acetylproduct schmilzt bei $122^{\circ}$, das Golddoppelsalz bei $203-204^{\circ}$. 
$0 \cdot 175 \mathrm{~g}$ des letzteren gaben $0 \cdot 0679^{\circ} \mathrm{Au}$.

$\underbrace{\text { Gefunden }}_{38 \cdot 30}$

Eine $8 \%$-ige Lösung zeigte bei $l=200, t=20, d \frac{20}{20}=1 \cdot 0144$ eine Ablenkung von $-3 \cdot 5^{\prime}$.

Somit geht auch die zweite Hälfte des Cinchonidin, geradeso wie jene von Cinchonin, Chinin und Chinidin, bei der Oxydation in Cincholoipon $\mathrm{C}_{9} \mathrm{H}_{17} \mathrm{NO}_{2}$ tiber, und dieses Spaltungsproduct der vier genannten Alkaloide hat unter allen Umständen auch dieselben optischen Eigenschaften.

Nach Beendigung dieser Versuche gelang es auch aus dem Schniderschitsch'schen Präparate, das nach mehrmonatlichem Stehen zu Krystallen erstarrt war, reichliche Mengen von Cincholoiponchlorhydrat zu isoliren. Über dessen Entstehung gilt dasselbe, was wir oben beim Chinidin (S. 2) bemerkt haben.

\section{Cinchonicin und Chinicin.}

Nachdem somit festgestellt ist, dass die bekannteren vier Chinaalkaloide neben der Chinolinreihe angehörigen Atomcomplexen einen zweiten mit neun Kohlenstoffatomen abspalten der chemisch und physikalisch in allen vieren derselbe ist, erschien es von Wichtigkeit, anch die amorphen Umwandlungsproducte der Chinaalkaloide in den Kreis der Untersuchung zu ziehen.

Es ist wohl nicht überflüssig, daran zu erinnern, dass das Cinchonidin stark links, das isomere Cinchonin stark rechts drehend ist, beide beim Erhitzen der Bisulfate leicht in das isomere, schwach rechts drehende Cinchonicin übergehen und dass unter analogen Bedingungen das links drehende Chinin ebenso wie das stark rechts drehende Chinidin in das schwach rechts drehende isomere Chinicin sich umlagern. Die chemischen Beziehungen der beiden genannten amorphen Umwandlungsproducte Cinchonicin und Chinicin zu ihren prächtig krystallisirenden Muttersubstanzen ist experimentell bis auf einen Versuch von Skraup, ${ }^{1}$ nach welchem das Cinchonicin ebenso Cinchoninsäure

1 Wiener Akad. Ber. 1879, Juliheft. 
liefert wie Cinchonin und Cinchonidin, kaum näher getreten worden. Eine Untersuchung der zwei amorphen umgelagerten Chinabasen hatte noch das besondere Interesse, dass sie die Möglichkeit bot, eine von Pasteur ${ }^{1}$ geäusserte Ansicht über die eigenthümlichen optiseben Verhältnisse der Chinaalkaloide zu controliren. Pasteur nahm an, den vier oft genannten krystallisirbaren Chinaalkaloiden sei ein und dieselbe schwach rechts drehende Gruppe gemeinsam, die in den links drehenden Alkaloiden mit einer stark links drehenden, in den rechts drehenden mit einer stark rechts drehenden zweiten Gruppe vereinigt sei, welche stark drehenden Gruppen bei der Umlagerung inactiv werden, während die Gruppen von schwächerem Rotationsvermögen ungeändert bleiben, darum die Umlagerungsproducte aus den Chinaalkaloiden von entgegengesetztem Drehungsvermögen optisch identisch sind, z. B. Cinchonin und Cinchonidin in dasselbe schwächer rechts drehende Cinchonicin tibergehen. Der Nachweis, dass die Alkaloide gleicher Zusammensetzung thatsächlich ganz dieselben Spaltungsproducte der Chinolinreihe geben, gibt jener Ansicht von vornherein einige Wahrscheinlichkeit.

Wir haben uns mit dem Chinicin beschäftigt, während Herrn Dr. Pu m die Untersuchung des Cinchonicins beschäftigt. ${ }^{*}$

Das Chinicin ist durch einstiindiges Erhitzen von Chininbisulfat auf $135^{\circ}$ dargestellt und das so erhaltene Product ohne weitere Reinigung zur Oxydation verwendet worden. Wir verfuhren ganz so, wie Sehniderschitsch mit dem Cinchonidin und der Eine von uns mit dem Chinidin, derart nämlich, dass der Oxydation mit Chromsäure eine solche mit Kaliumpermanganat vorausging. Der Verlauf der weiteren Operationen war genau derselbe, wie bei den anderen Chinabasen. Neben reichlichen

1 Jahresber. f. 1853474.

2 Nachdem Jungfleisch (Compt. rend. 105, 1255 u. ff.) festgestellt hat, dass das Cinchonin durch Erhitzen eine grosse Zahl von Umlagerungsproducten zu liefern vermag, könnte die Eigenart des Cinchonicins und auch des Chinicins angezweifelt werden. Wir halten sie jedoch für genügend sichergestellt, da ein so scharfer Beobachter wie Hesse die Umlagerungsproducte mit genau denselben chemischen und optischen Eigenschaften sowohl aus den links- als den rechtsdrehenden Alkaloiden der Chinarinde erhalten hat. 
Mengen von Chininsäure konnten wil wiederum ein in Alkohol nicht, in Wasser leicht lösliches amorphes Bleisalz, aus diesem ohne besondere Schwierigkeit eine Substanz von der Zusammensetzung und den Eigenschaften des Cincholoiponsäurechlorhydrats and weiter eine in Alkohol lösliche Fraction isoliren, aus der nach umständlicher, Seite 4 etwas näher beschriebenen Reinigung mit Quecksilberchlorid Cincholoiponchlorbydrat auskrystallisirte.

Es sei bemerkt, dass die Reinigung des Cincholoiponehlorhydrats wesentlich leichter ist, wenn man die syrupösen Lösungen, dieCincholoipon enthalten, von vornherein nicht mit festem Quecksilberchlorid, sondern mit einer kochend gesättigten Lösung desselben ausfällt, wieder eindampft, abermals ausfällt und dies so lange fortsetzt, bis nach weiterem Zusatze keine Fällung mehr eintritt. Die von uns isolirten zwei Substanzen wichen in keiner Weise von den Spaltungsproducten der vier Chinabasen ab. Das Cincholoiponsä̈rechlorhydrat verflïssigte sich bei $193^{\circ}$, mitSilberoxyd zerlegt gab es die charakteristischen Tafeln der freien Cincholoiponsäure.

$0 \cdot 1557 \mathrm{~g}$ gaben $0 \cdot 0995 \mathrm{AgCl}$.

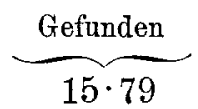

$$
\frac{\text { Berechnet }}{15 \cdot 88}
$$

Die $4 \%$-ige Lösung zeigte bei $d \frac{20}{20}=1 \cdot 0131, l=200$, $t=20$ eine Ablenkung von $\alpha_{D}=+2^{\circ} 51^{\prime}$, was einem sp. D. von $[\alpha]_{D}=+35 \cdot 61^{\circ}$ entspricht, Zahlen, welchen von jenen, die für dieselbe Substanz aus den krystallisirten Chinabasen ermittelt wurden, so gut wie nicht abweichen.

Das Cincholoiponchlorhydrat verflitssigte sich bei $199-201^{\circ}$.

$0 \cdot 215 \mathrm{~g}$ Substanz gaben $0 \cdot 1467 \mathrm{AgCl}$.
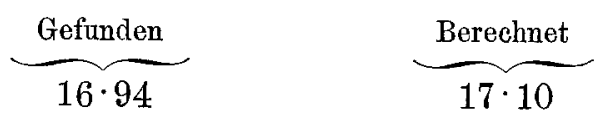

Sein Goldsalz schmilzt bei $202-204^{\circ}$, das Acetylproduct bei $122^{\circ}$, beide zeigen die gewöhnlichen Formen. 
Die Menge des Chlorhydrates genïgte nur zur Bereitung des gentigenden Volumens einer $5 \%$-igen Lösung. Diese gab eine Ablenkung von $-2^{\prime}$ die gleichfalls fast völlig mit der des Cincholoiponchlorhydrats anderer Herkunft übereinstimmt.

Die Zerfallsproducte des Chinicins sind also chemisch und physikalisch vollständig identisch mit jenen des Chinins und Chinidins.

Wir können dem zufügen, dass Herr Dr. Pam zu ähnlichen Resultaten betreffs des Cinchonicins gelangte, wenigstens ist heute schon sichergestellt, dass aus demselben Cincholoiponsäure, in jeder, auch in optischer Beziehung völlig identisch mit der Verbindung aus Cinchonin, Cinchonidin ete., entsteht. Ob das Cinchonicin auch Cincholoipon abspaltet, ist noch nicht ausser allem Zweifel, da die Behandlung jener Fraction, in welcher es zu suchen ist, Schwierigkeiten verursacht, doch nach den bisherigen Beobachtungen mehr als wahrscheinlich.

Hält man alle in dieser und den vorhergehenden Mittheilungen gemachten Erfahrungen zusammen, so gelangt man zu dem Schlusse, dass nicht nur die vier natürlichen Chinaalkaloide, Chinin, Chinidin, Cinchonin und Cinchonidin, sondern auch ibre amorphen Umlagerungsproducte Chinicin und Cinchonicin in ihrer Constitution so ähnlich sind, dass die Untersuchung ihrer Spaltungsproducte keine anderen Differenzen aufdecken konnte, als solche schon aus der empirischen Formel hervorgehen.

Alle sechs Basen enthalten einen und denselben Rest, jenen, der bei der Oxydation als Cincholoipon abgespalten wird und der optisch activ ist. Wenn auch die drehende Kraft des Cincholoipons eine äusserst geringe ist - die directen Ablesungen fallen innerhalb der Fehlergrenze - so ist doch einmal auffallend, dass bei verschiedenen Substanzen und von zwei verschiedenen Beobachtern die Ablenkung immer in demselben Sinne nach links gefunden worden ist, und noch beweisender ist, dass die Cincholoiponsäure, die zweifellos als Spaltungskörper des Cincholoipons in Betracht kommt, ganz deutlich dreht.

Da das freie Cincholoipon bisher optisch nicht untersucht ist und verschiedene Beispiele zeigen, dass Verbindungen activer Substanzen oft im entgegengesetzten Sinne drehen, wie die unver- 
bundenen activen Körper, kann man das optische Drehungsvermögen der zwei Spaltungsproducte nicht vergleichen. Selbst aber, wenn das Cincholoipon in freiem Zustande und vielleicht auch in seinen anderen Verbindungen linksdrehend sein sollte, muss es doch eine Atomgruppirung enthalten, die eine Rechtsdrehnng anstrebt, da es bei weiterer Oxydation in die rechtsdrehende Cincholoiponsäure übergeht.

Die Pasteur'sche Hypothese ist also experimentell soweit ansser Zweifel gestellt, als sie sich auf die eine, den Chinaalkaloiden gemeinschaftliche Gruppe desselben optischen Drehungsvermögens bezieht.

Damit wird ihr zweiter Theil, welcher in den isomeren Paaren optisch entgegengesetzt wirkende Gruppen annimmt, in hohem Grade auch vom chemischen Standpunkte wahrscheinlich, dadurch aber für die Beurtheilung der Constitution der Alkaloide ein weiterer Anhaltspunkt geboten.

Wenn jene Gruppen, die als Chinolinderivate abspaltbar sind, optisch wirksam sein sollen, mïssen sie mindestens ein asymmetrisches Kohlenstoffatom enthalten, während sowohl Cinchoninsäure als Chininsäure im Lichte der üblichen Chinolinformel ein solches nicht besitzen, also nach der Le Bel, van-t-Hoff'schen Theorie optisch inactiv sein müssen.

In optischer Beziehung sind unseres Wissens die genannten zwei Säuren bisher nicht untersucht. Die Chininsäure, von der wir gentigende Mengen ganz reiner Substanz besassen, haben wir als thatsächlich inactiv gefunden, so bei Anwendung einer mehr als $15 \%$-igen Lösung des Natriumsalzes in Wasser und einer etwa $8 \%$-igen Lösung in wässerig alkoholischer Salzsäure Damit erscheinen alle Vermuthungen, die auf Grund der Chinolinformel mit Parabindung zwischen Stickstoff und Kohlenstoff anzustellen wären, wohl überflïssig.

Der Widerspruch zwisehen der Voraussetzing, dass die "Chinolinhälfte" der Alkaloide optiseh wirksam ist, und der Thatsache, dass die abgespaltenen Chinolinderivate es aber nicht sind, ist durch die Annahme lösbar, das Carboxylkohlenstoffatom der Chininsäure und Cinchoninsäure, vermittele die Verknüpfung beider Alkaloidhälften, es sei im unverletzten Molekül asymmetrisch und verliere die Asymmetrie bei der Spaltung. 
Diese Asymmetrie kann selbstverständlich in der verschiedenartigsten Art begriundet sein, immerhin sind bedingsweise Vermuthungen über deren Ursachen zulässig.

Einmal wird die structurmässige Verknüpfung bei diesem asymmetrischen Kohlenstoffatome eine ziemlich bewegliche, jedenfalls eine beweglichere sein miissen, als bei jenem oder jenen im Cincholoiponreste, denn bei Temperaturerhöhung tritt bei ersterem stereochemische Umlagerung ein, bei letzteren nicht.

Das andere Mal kann man einige Arten der Bindung für das verknüpfende Kohlenstoffatom ausschliessen, so $-\mathrm{CH}_{2}-$ oder $-\mathrm{CO}-$; ferner ist sicher, dass, wenn es nicht mit einem Hydroxyl oder einer analogen Atomgruppe in Verbindung steht, es mindestens mit drei Valenzen an Kohlenstoff oder Stickstoff gebunden, der Cincholoipon liefernde Rest, die sogenannte zweite Hälfte, mit ihm also mindestens zweifach verknüpft sein muss. Selbstverständlich kann eine solche dreifache Bindung noch immer in Betracht kommen, wenn das asymmetrische Kohlenstoffatom ein Hydroxyl bindet. Wir erwähnen diesen Punkt hauptsächlich desshalb, weil uns vorkommt, dass diese Constitutionsverhältnisse bei richtiger Erkenntniss in befriedigender Weise erklären werden, warum Königs und der Eine von uns bisher zu so verschiedenen Ansichten über die "zweite Hälfte" der Chinabasen gelangt sind.

Die Beziehungen der wichtigeren Chinaalkaloide untereinander lassen sich mit wenigen Worten dahin zusammenfassen, dass Chinin, Chinidin und Chinicin aller Wahrscheinlichkeit nach structuridentisch, und stereochemisch isomer sind, ebenso Cinchonidin, Cinchonin und Cinchonicin. Die ersten Glieder der zwei Gruppen sind den Linksweinsäuren analog, die folgenden den Rechtsweinsäuren, die letzten der optisch nichtactiven Weinsäure vergleichbar, vermuthlich der sogenannt inactiven. Die Analogie ist nur annähernd, denn die optische Activität verschwindet bei den Chinaalkaloiden durch die Umlagerung nicht vollständig.

Die Chinaalkaloide enthalten mindestens zwei, aller Wahrscheinlichkeit nach mehr asymmetrische Kohlenstoffatome, fuir letzteres sprechen unter Anderem die neuen vier Umlagerungs- 
230 Zd. H. Skraup u. J. Würstl, Constitution der Chinaalkaloide.

producte des Cinchonins, die Jungfleisch und Léger (Compt. rend. 105, 1257 u. ff.) erhalten haben. Diese complicirten Verhältnisse geben auch dafür Erklärung, dass, wie Hesse gefunden hat, das Drehungsvermögen vom Chinicin und Cinchonicin vom arithmethischen Mittel der Constanten vom Chinin und Chinidin und rom Cinchonidin und Cinchonin, so erheblich abweicht. Die Untersuchung der Chinaalkaloide wird fortgesetzt. 